\title{
Microbubbles and Electromagnetic waves for the treatment of angiogenesis-dependent human ailments
}

\section{Pegah Moradi \\ School of Physics, Universiti Sains Malaysia, Penang, Malaysia}

Please cite this article:

Pegah Moradi. (2017). Microbubbles and Electromagnetic waves for the treatment of angiogenesis-dependent human ailments Angiotherapy, 1(1), pages 018-021.

Significance | Electromagnetic waves are succesfully utilized for the treatment of angiogenic diseases and diagnosis.

\section{Graphical Abstract}

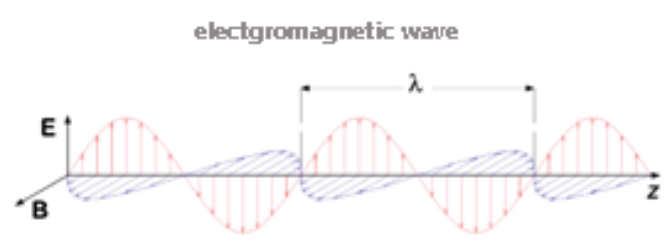

Ultrasound, angiogenesis 8 Therapeutic efficacy

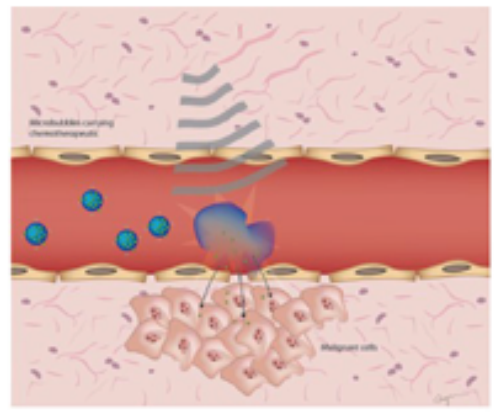

cancer 8 angiogenesis

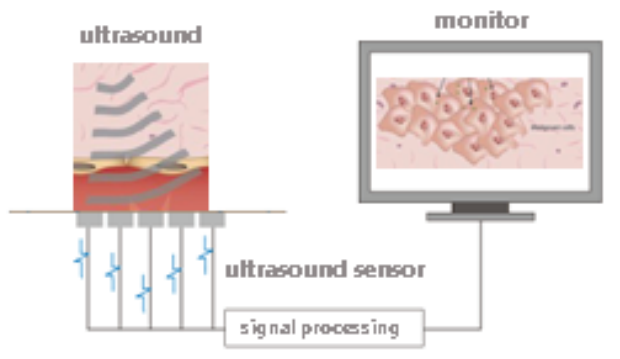

signal procesing

${ }^{*}$ Correspondence: School of Physics, Universiti Sains Malaysia, Penang, Malaysia. E-mail: med.physics64@yahoo.com 


\title{
Microbubbles and Electromagnetic waves for the treatment of angiogenesis-dependent human ailments
}

\author{
Pegah Moradi
}

\begin{abstract}
Molecular imaging such as ultrasound is capable of clarifying the expression of angiogenic markers in vivo. However, the capability of the method for volumetric "multitarget quantification" and for the test of anti-angiogenic therapy response has been investigated partly. Histologic data indicates that high concentrations of VEGFR-2 and of av $\beta 3$ integrin during the growth of tumours are associated with an upregulation of the densities of markers by the endothelial cells, while a down-regulation of during therapy is due to decreasing relative vessel density. Therefore, targeted ultrasound appears practical for the longitudinal molecular profiling of tumor angiogenesis and for the sensitive evaluation of therapy effects in vivo.
\end{abstract}

Keywords: tumour angiogenesis, VEGF ultrasound, contrast enhanced ultrasound

Abbreviations: VEGF, vascular endothelial growth factor

\section{Biology of angiogenesis}

Angiogenesis, the process of new blood vessel formation, plays central role in tumour neovascularisation. Angiogenesis is needed to bring oxygen and other nutrients and to remove cellular waste residues. (Folkman, 1995; Zhu et al., 2011; Carmeliet and Jain, 2000).

Significance | Electromagnetic waves are succesfully utilized for the treatment of angiogenic diseases and diagnosis.

${ }^{*}$ Correspondence: School of Physics, Universiti Sains Malaysia, Penang, Malaysia E-mail: med.physics64@yahoo.com

Edited by Md Shamsuddin Sultan Khan, Hawkesbury Institute for the Environment, University of Western Sydney, Hawkesbury Campus, Bourke Street, Richmond, NSW AUSTRALIA 2753 and accepted by the Editorial Board April 4, 2017 (received for review February 19, 2017)
Neo-angiogenesis is a key step in the initiation of tumours and can be a predicator of cancer metastasis to other organs. Recent studies in cellular and molecular biology suggest identifying new angiogenic biomarkers and their of vascular endothelial growth factor (Duda, et al., 2007) and its receptor tyrosine kinase (Young and Dyson, 1990), VEGFR-1 (Kwok et al. 2013) and VEGFR-2 (Duda, et al., 2007; Fujii et al., 2013; Kadambi et al., 2001; Tsuzuki et al., 2000; Hicklin and Ellis, 2005) on new tumour blood vessels formation.

Binding of VEGF to its receptor induces a signalling cascade that enhances the proliferation, migration and survival of endothelial cells and finally leads to angiogenesis. Primarily, the angiogenic effects of the VEGF family are mediated by VEGF-A which is the most-characterized signalling pathway in developmental and tumor angiogenesis (Selleck, 2006; Bagri, 2010; Tortora, 2008; Grothey, 2009).

The VEGF/VEGFR signalling pathway plays an effective role in both normal vasculature development and many other disease processes. The VEGF family has seven members. All members have VEGF homology domain: VEGF-A, VEGF-B, VEGF-C, VEGF-D, VEGF-F and placenta growth factor (Cai and Chen, 2006; Ferrara, 2004).

It has been reported that the isoforms have VEGF-A . This homodimeric, disulfide-bound glycoprotein has different amino-acid residues, such as VEGF121 and VEGF165. Apart from the difference in molecular weights, these isoforms differ in their biologic properties in terms of their ability to bind to cell surface heparin sulphate proteoglycans (Ferrara, 2004).

VEGFR-1 is the basis of most physiologic and developmental angiogenesis, and as the stage of development changes, its activity

Author Affiliation:

School of Physics, Universiti Sains Malaysia, Penang, Malaysia

\section{Please cite this article:}

Pegah Moradi. (2017). Microbubbles and Electromagnetic waves for the treatment of angiogenesis-dependent human ailments Angiotherapy, 1(1), pages 018-021. 
cell type in which it is expressed and the physiological and pathological conditions change accordingly. VEGFR-2 is the mixture of mitogenic and angiogenic effects of VEGF. The main role of the VEGF/VEGFR signalling pathway in cancer treatment is confirmed by the approval of the humanized anti-VEGF monoclonal antibody evacizumab (Sun et al.,2005; Watanabe et al., 2004; Prewett et al., 1999). Such a therapeutic role has increased the necessity of scanning the VEGF/VEGFR imaging with targeted angiogenic therapy in last few years.

\section{Ultrasound}

One of the usages of Ultrasound is exposing the dynamic flow of vessels of large, usually less than $500 \mu \mathrm{m}$, in diameter. This unfortunately means the imaging resolution cannot distinguish angiogeneic capillaries. For this purpose two ultrasound modes can be combined, gray-scale and Power Doppler. Therefore, Doppler ultrasound can better determine the flow and flow resistance in native vasculature and vasculogenesis, which means that additional mature vessels have been recruited to the tumor (Halpern and Strup, 2000).

Much research has been conducted on benign and malignant lesion. Analysing the spectral wave pattern can determine blood velocity, direction and downstream resistance. The function of Resistive Index (RI) is to indicate the diastolic vascular resistance in a tumour. However, low vascular resistance and high vascular resistance are concerned with the lack of vasomotor in low and high oncotic interstitial pressures, respectively. Hence, the heterogeneous nature of tumour vascularity may show both high and low RI values in the same lesion. However, there can be a notable overlap of RIs between benign and malignant lesions (Cosgrove , 2014). RI measurements are related to microvessel density (MVD) in breast, brain and ovarian cancer, but the connection is indirect since the microvessels are significantly below the spatial resolution of ultrasound (Turkbey et al., 2009). Contrast-enhanced ultrasound using microbubbles can detect images from smaller vessels and facilitate the assessment of patient response to anti-angiogenesis treatment in some tumour types. However, due to some constraints in spatial resolution, operator dependence, the short time window for imaging, and the limited field or view (Hata et al., 1998; Yang et al., 2002; Alcazar, 2006) this type of ultrasound has not been extensively used.

\section{Ultrasound Imaging of VEGFRs and Endostatin}

Some features (e.g.safety, low cost, ease of use, and wide availability) (Bloch et al., 2004) of ultrasound (ultrasonography) has made it the most commonly used clinical imaging system. The contrast of ultrasound depends on four major parameters, such as sound speed, sound attenuation, imaging algorithm, and backscatter (Wink et al., 2006). Ultrasound can be used to depict the microcirculation with two methods of Doppler and micro- bubble methods (Liang and Blomley, 2014).

The power of Doppler signal can be quantified to provide an estimate of relative fractional vascular volume, whereas microbubbles can show blood flow down to the microcirculation level by increasing the signal from smaller vessels. Non-invasive ultrasound imaging which emphasize results like DCE-MRI , can be obtained during tumour development or anti-angiogenesis treatment. The results are comparable with ex vivo histologic data (Drevs et al., 2000; Forsberg et al., 2004; Forsberg et al., 2002; Cheung et al., 20070. However, the data have not shown non-invasive direct imaging of VEGF/VEGFR expression and only new ultrasound imaging of VEGF/VEGFR expression was not reported (Korpanty et al., 2007).

Contrast-enhanced ultrasound (Kittel et al., 1976) imaging usually uses microbubbles, that are roughly a micrometer in size. This means that only the tumour endothelium can be targeted, since these microbubbles are too large to extravasate. In a mouse model of pancreatic cancer, targeted microbubbles were utilized to reflect and calculate the vascular effects of two anti-tumour therapies on both subcutaneous and orthotopic pancreatic tumours (orpanty et al., 2005).

Tumour-bearing mice were treated with anti-VEGF, gemcitabine and the place of antibody-conjugated microbubbles to VEGFR-2-e xpressing or VEGF-activated blood vessels was detected by CEU. Signal intensity of the tumour vasculature was significantly increased with the targeted microbubbles compared with untargeted or control IgG-conjugated microbubbles. The video signal intensity from the targeted microbubbles under therapy was also variable with the level of expression of the target (either VEGFR-2 or the VEGF-VEGFR complex), and with the density of the tumour vascularization. In another study, VEGFR-2 expression in two murine tumour models was imaged with microbubbles technique. The microbubbles were conjugated to anti-VEGFR-2 monoclonal antibodies (Willmann et al., 2008).

The targeted microbubbles produced a remarkably higher average video signal intensity compared to control microbubbles in both tumour models. Whereas, the video signal intensity was also lower when anti-VEGFR-2 antibodies (blocking antibodies) were used, to show target specificity. These reports show that targeted microbubbles can be a unique and attractive tool for the imaging of tumour angiogenesis, like for the in vivo demonstrating of vascular effects after therapy. Ultrasound has a high spatial resolution in range of $50-500 \mu \mathrm{m}$, however it also has some disadvantages, such as relatively poor tissue penetration (generally a few centimetres, depends on the frequency used) and limited sensitivity (Massoud and Gambhir, 2003).

In addition, further development of molecular imaging with ultrasound might allow molecular imaging of a greater number of diseases, which will advance in technology for ligand attachment 
to microbubbles, greater characterization of the acoustic behaviour of targeted contrast agents, and more sensitive methods. Acoustic failure of "payload-bearing" microbubbles has been used to deliver drugs or to adjoin gene transfection (Shohet et al., 2000). Thus, angiogenesis-targeted microbubbles may also have future applications in site-specific cancer therapy(Young and Dyson, 1990).

Cationic liposomes consist of plasmid DNA attached to the phospholipid shell of gas-filled microbubbles $2-4 \mu \mathrm{m}$ in diameter. The microbubble-liposome complexes are infused intravenously (i.v.) and destructed within the myocardial microcirculation by low-frequency ultrasound (Korpanty et al., 2005).

The targeted endostatin microbubbles, complexed with focused, directional ultrasound radiation can destroy tumour microvasculature of subcutaneous colon translocation in situ colon cancer, so inhibits the tumour angiogenesis (Kwok et al., 2013). Ultrasound-targeted microbubble destruction (UTMD) can be utilized to deliver silencing gene therapy to tumours. Researchers hypothesized that UTMD would be effective in suppressing angiogenesis within tumours, and that the magnitude of target knockdown will be affected by the modulation of the ultrasound pulsing intervals (Korpanty et al., 2005) during UTMD.

UTMD of vascular endothelial growth factor receptor-2 (VEGFR2) short hairpin (sh)RNA plasmid in an heterotopic mammary adenocarcinoma model in rats was used to evaluate PIs of 2, 5, 10, and 20 seconds. The findings that UTMD with a PI of 10 seconds led to a more efficient knockdown of VEGFR2 by PCR, immunostaining, western blotting as well as smaller tumor volumes and perfused areas, and lower tumour microvascular blood volume (MBV) and flow by contrast-enhanced ultrasound (Kittel et al., 2010) relative to UTMD-treated tumours at 2, 5, and 20 seconds, control tumours, tumours treated with intravenous shRNA plasmid and scrambled plasmid.

CEU perfusion assessment using a therapeutic probe showed that tumors were fully replenished with microbubbles within 10 seconds, but incompletely replenished at PI-2 and PI-5 seconds. Finally, for anti-VEGFR2 cancer gene therapy by UTMD, PI of 10 seconds leads to higher target knock down and a more anti-angiogenic effect. Complete replacing of tumour vasculature with silencing gene-bearing microbubbles in between destructive pulses of UTMD is needed to maximize the efficacy of anti-angiogenic cancer gene therapy (Fujii, H., et al., 2013).

\section{Contrast-enhanced ultrasound}

Contrast-enhanced ultrasound (Kittel et al., 2010) techniques are based on the ultrasonic detection of microbubble contrast agents during their microvascular transit. The techniques have been developed for the molecular imaging of the vascular phenotype as well as the characterization of angiogenesis by utilizing novel site-targeted micro bubbles. These molecular imaging techniques have the potential to further characterize the angiogenic response. Its aim is to optimize gene- and cell-based strategies for therapeutics targetting neovascularisation, and finally serve to show the therapeutic effects in patients registered in clinical trials of regenerative therapies (Leong-Poi, 2009).

CEU methods estimate tissue perfusions which utilize free-flowing non-targeted microbubbles, the method applies custom-designed microbubble contrast agents that are in regions of disease by virtue of their shell composition or by the conjugation of specific targeting ligands on their outer surface. Since microbubbles stay completely within the intravascular space throughout their circulation, the processes that can be targeted are characterized by processes that occur primarily within the vascular compartment like atherosclerosis, ischemia, and inflammation (Dewitte et al., 2011).

The ability to distinguish the first molecular changes that happen in angiogenesis, before changes in perfusion or vascular morphology, would allow early monitoring of therapies. In pre-clinical studies, such techniques could also produce data on the physiological responses to angiogenic and cell-based therapies, which clarifies future insight into mechanisms related to the pro-angiogenic response.

\section{Conclusion}

In conclusion, molecular ultrasound is a hopeful quantitative technology for imaging of multiple angiogenic markers. It prepares a high sensitivity as contrast agents, excellent biocompatibility, and meaningful potential for clinical translation. It is suitable for the identification of angiogenic markers as well as the evaluation of anti-angiogenic therapy effects. Thus, utilizing molecular ultrasound suggests multiple options to develop biomedical research in the future.

\section{Acknowledgment}

The authors would like to express their gratitude to University Science Malaysia for research grant (no.131/0210/0350) and facilities.

\section{Author Contribution}

Pegah M. made substantial contributions to the conception design of the manuscript, and review of the literature.

\section{Competing financial interests}

The author(s) declare no competing financial interests.

\section{References}

Alcazar, J.L. (2006). Tumor angiogenesis assessed by three-dimensional power Doppler ultrasound in early, advanced and metastatic ovarian cancer: a preliminary study. Ultrasound in obstetrics \& gynecology, 28(3), 325-329.

Bagri, A., et al. (2010). Use of anti-VEGF adjuvant therapy in cancer: challenges and rationale. Trends in molecular medicine, 16(3), 122-132.

Babes, L., et al. (1999). Synthesis of iron oxide nanoparticles used as MRI contrast agents: a parametric study. Journal of colloid and interface science, 212(2), 474-482.

Bloch, S.H., P. Dayton, and K.W. Ferrara. (2004). Targeted imaging using ultrasound 
contrast agents. Engineering in Medicine and Biology Magazine, IEEE, 23(5), 18-29.

Cai, W. and X. Chen. (2006). Multimodality imaging of vascular endothelial growth factor and vascular endothelial growth factor receptor expression. Frontiers in bioscience: a journal and virtual library, 12, 4267-4279.

Cosgrove, D. (2014). Angiogenesis imaging-ultrasound. The British journal of radiology.

Cheung, A.M., et al. (2007). Detecting vascular changes in tumour xenografts using micro-ultrasound and micro-CT following treatment with VEGFR-2 blocking antibodies. Ultrasound in medicine \& biology, 33(8), 1259-1268.

Carmeliet, P. and R.K. Jain,. (2000). Angiogenesis in cancer and other diseases. nature, 407(6801), 249-257.

Chi, A.S., et al. (2009). Angiogenesis as a therapeutic target in malignant gliomas. The oncologist, 14(6), 621-636.

Drevs, J., et al. (2000). Effects of PTK787/ZK 222584, a specific inhibitor of vascular endothelial growth factor receptor tyrosine kinases, on primary tumor, metastasis, vessel density, and blood flow in a murine renal cell carcinoma model. Cancer Research, 60(17), 4819-4824.

Dewitte, H., et al. (2011). The use of ultrasound contrast agents in cancer immunotherapy: The use of mRNA-loaded microbubbles for transfection of dendritic cells. Parallel HOW TO session.

Duda, D.G., et al. (2007). VEGF-targeted cancer therapy strategies: current progress, hurdles and future prospects. Trends in molecular medicine, 13(6), 223-230.

Ferrara, N. (2004). Vascular endothelial growth factor: basic science and clinical progress. Endocrine reviews, 25(4), 581-611.

Folkman, J. (1995). Angiogenesis in cancer, vascular, rheumatoid and other disease. Nature medicine, 1(1), 27-30.

Forsberg, F., et al. (2004). Assessment of angiogenesis: implications for ultrasound imaging. Ultrasonics, 42(1), 325-330.

Forsberg, F., et al. (2002). Comparing contrast-enhanced ultrasound to immunohistochemical markers of angiogenesis in a human melanoma xenograft model: preliminary results. Ultrasound in medicine \& biology, 28(4), 445-451.

Fujii, H., et al. (2013). Optimization of ultrasound-mediated anti-angiogenic cancer gene therapy. Molecular Therapy—Nucleic Acids, 2(5), e94.

Grothey, A. and E. Galanis. (2009). Targeting angiogenesis: progress with anti-VEGF treatment with large molecules. Nature reviews Clinical oncology, 6(9), 507-518.

Hicklin, D.J. and L.M. Ellis. (2005). Role of the vascular endothelial growth factor pathway in tumor growth and angiogenesis. Journal of clinical oncology, 23(5), 1011-1027.

Halpern, E.J. and S.E. Strup. (2000). Using gray-scale and color and power Doppler sonography to detect prostatic cancer. American Journal of Roentgenology, 174(3), 623-627.

Hata, K., et al. (1998). Expression of thymidine phosphorylase in malignant ovarian tumors: correlation with microvessel density and an ultrasound-derived index of angiogenesis. Ultrasound in obstetrics \& gynecology, 12(3), 201-206.

Kwok, S.J., et al. (2013). Ultrasound-mediated microbubble enhancement of radiation therapy studied using three-dimensional high-frequency power doppler ultrasound. Ultrasound in medicine \& biology, 39(11), 1983-1990.

Kadambi, A., et al. (2001). Vascular Endothelial Growth Factor (VEGF)-C Differentially Affects Tumor Vascular Function and Leukocyte Recruitment Role of VEGF-Recep- tor 2 and Host VEGF-A. Cancer research, 61(6), 2404-2408.

Korpanty, G., et al. (2007). Monitoring response to anticancer therapy by targeting microbubbles to tumor vasculature. Clinical Cancer Research, 13(1), 323-330.

Kittel, C., P. McEuen, and P. McEuen. (1976). Introduction to solid state physics. Vol. 8, Wiley New York.

Korpanty, G., et al. (2005). Targeting vascular endothelium with avidin microbubbles. Ultrasound in medicine \& biology, 31(9), 1279-1283.

Korpanty, G., et al. (2005). Targeting of VEGF-mediated angiogenesis to rat myocardium using ultrasonic destruction of microbubbles. Gene therapy, 12(17), 1305-1312.

Leong-Poi, H. (2009). Molecular imaging using contrast-enhanced ultrasound: evaluation of angiogenesis and cell therapy. Cardiovascular research, 84(2), 190-200.

Liang, H. and M. Blomley. (2014). The role of ultrasound in molecular imaging. The British journal of radiology.

Massoud, T.F. and S.S. Gambhir. (2003). Molecular imaging in living subjects: seeing fundamental biological processes in a new light. Genes \& development, 17(5), 545-580. Prewett, M., et al. (1999). Antivascular endothelial growth factor receptor (fetal liver kinase 1) monoclonal antibody inhibits tumor angiogenesis and growth of several mouse and human tumors. Cancer research, 59(20), 5209-5218.

Selleck, S.B. (2006). Signaling from across the way: transactivation of VEGF receptors by HSPGs. Molecular cell, 22(4), 431-432.

Sun, J., et al. (2005). Inhibiting angiogenesis and tumorigenesis by a synthetic molecule that blocks binding of both VEGF and PDGF to their receptors. Oncogene, 24(29), 4701-4709.

Shohet, R.V., et al. (2000). Echocardiographic destruction of albumin microbubbles directs gene delivery to the myocardium. Circulation, 101(22), 2554-2556.

Tsuzuki, Y., et al. (2000). Vascular endothelial growth factor (VEGF) modulation by targeting hypoxia-inducible factor-1 $\alpha \rightarrow$ hypoxia response element $\rightarrow$ VEGF cascade differentially regulates vascular response and growth rate in tumors. Cancer research, 60(22), 6248-6252.

Tortora, G., F. Ciardiello, and G. Gasparini. (2008). Combined targeting of EGFR-dependent and VEGF-dependent pathways: rationale, preclinical studies and clinical applications. Nature Clinical Practice Oncology, 5(9), 521-530.

Turkbey, B., et al. (2009). Imaging of tumor angiogenesis: functional or targeted? AJR. American journal of roentgenology, 193(2), 304.

Watanabe, H., et al. (2004). Anti-vascular endothelial growth factor receptor-2 (Flk-1/KDR) antibody suppresses contact hypersensitivity. Experimental dermatology, 13(11), 671-681.

Wink, M.H., et al. (2006). Ultrasound imaging and contrast agents: A safe alternative to MRI? Minimally Invasive Therapy \& Allied Technologies, 15(2), 93-100.

Willmann, J.r.K., et al. (2008). US Imaging of Tumor Angiogenesis with Microbubbles Targeted to Vascular Endothelial Growth Factor Receptor Type 2 in Mice 1. Radiology, 246(2), 508-518.

Yang, W.T., et al. (2002). Correlation between color power Doppler sonographic measurement of breast tumor vasculature and immunohistochemical analysis of microvessel density for the quantitation of angiogenesis. Journal of ultrasound in medicine, 21(11), 1227-1235.

Young, S. and M. Dyson. (1990). The effect of therapeutic ultrasound on angiogenesis. Ultrasound in medicine \& biology, 16(3), 261-269.

Zhu, A.X., et al. (2011). HCC and angiogenesis: possible targets and future directions. Nature reviews Clinical oncology, 8(5), 292-301. 